\title{
Teaching PLCs with the IEC 61131 Standard Languages
}

\author{
James Rehg, Bruce Muller \\ Penn State Altoona
}

\begin{abstract}
Programmable Logic Controllers are the de facto standard for sequential control of discrete automation systems and are fast becoming the system of choice for control of analog process systems. The new languages introduced in the IEC 61131 PLC standard will increase the number of applications suitable for PLC control. This paper provides a description of the new standard and describes a laboratory at Penn State Altoona that supports a two course sequence for PLC. The laboratory has 16 student stations and 4 automated assembly systems linked by an Ethernet LAN with a DeviceNet sub-network. The laboratory supports ladder logic programming using SLC 500 system technology for an introduction to PLC concepts taught in the first course. The second course includes programming in five of the IEC 61131 languages (ladder logic, structured text, function block diagrams, and sequential function chart languages) on an automated assembly system using a DeviceNet LAN and DeviceNet compatible control devices. In addition, the paper describes how the different languages were used and introduced.
\end{abstract}

\section{Introduction to the IEC 61131 PLC Standard}

The first standard for PLCs was published in 1978 by the National Electrical Manufacturers Association (NEMA). However, the rapid growth in PLCs across national boundaries demanded a broader standard. In 1979 the International Electrotechnical Commission (IEC) established a working group to look at the complete standardization of PLCs ${ }^{1}$. The PLC standard, called IEC 1131, (changed later to IEC 61131) has the following parts.

- Part 1: General information establishes the definitions and identifies the principal characteristics relevant to the selection and application of programmable controllers and their associated peripherals.

- Part 2: Equipment requirements and tests specifies equipment requirements and related tests for programmable controllers (PLC) and their associated peripherals.

- Part 3: Programming languages defines, as a minimum set, the basic programming elements, syntactic and semantic rules for the most commonly used programming languages, as well as major fields of application, applicable tests and means by which manufacturers may expand or adapt those basic sets to their own programmable controller implementations. Five PLC languages are defined.

- Part 4: User guidelines a technical report providing general overview information and application guidelines of the standard for the end user of programmable 
controllers.

- Part 5: Messaging service specification defines the data communication between programmable controllers and other electronic systems using the Manufacturing Message Specification (MMS, according to International Standard ISO/IEC 9506.

- Part 7: Fuzzy control programming defines basic programming elements for fuzzy logic control as used in programmable controllers.

- Part 8: Guidelines for the application and implementation of programming languages provides a software developers guide for the programming languages defined in Part 3.

Part 6 is not present since it is reserved for future use. Part three of the standard, Programming Languages for Programmable Controllers, was released in 1993 and specifies the standards for PLC software. Part three covers PLC configuration, programming and data storage. The IEC 61131-3 standard provides a very specific and detailed definition of PLC programming. The programming standard specifies five languages that PLC vendors should support if their controller is specified as IEC 61131 compatible. The languages are:

- Ladder Diagrams (LD)

- Sequential Function Charts (SFC)

- Function Block Diagrams (FBD)

- Structured Text (ST)

- Instruction List (IL)

The PLC languages used in specific countries or continents vary greatly. German programmers prefer Instruction List and Structured Text; while many of the PLC experts in France structure their programs using Sequential Function Charts. In the US, a combination of SFC and vendor specific ladder logic is the favorite programming platform. The IEC 61131-3 languages and the US vendor specific languages cannot be integrated directly because each have features not found in the other. Since vendor specific ladder logic is used almost exclusively with the SFC providing programming structure, many features have been added to the ladder syntax. The US vendor ladder implementation not only has Boolean expressions, as in IEC 61131-3 Ladder Diagrams, but has programming algorithms for many data types and many functions. While few US manufacturers program PLC solutions in ST, ST is used frequently inside of the SFC structure. Function Block Diagrams are finding increased use in US industries for process control and in motion control with servo and non-servo drive applications.

\section{PLC Course Sequence}

The four-year Electromechanical Engineering Technology program at Penn State Altoona has a two course sequence to teach programmable logic controllers. The goals for each course are summarized below.

EET 220 Programmable Logic Controllers - 2 credits (1 hour lecture and 2 hours laboratory) 
- Introduce students to the PLC program structure using ladder logic syntax in the Allen Bradley RS Logix 500 language on a SLC 505 PLC system

- $\quad$ Cover the PLC command structure used in a majority of the PLC programs developed in industry including commands from: bit, timer/counter, compare, compute/math, move/logical, and program control command groups

- Execute programs using only push button and selector switches for input and lights for the outputs (see Figure 1 for view of student programming station)

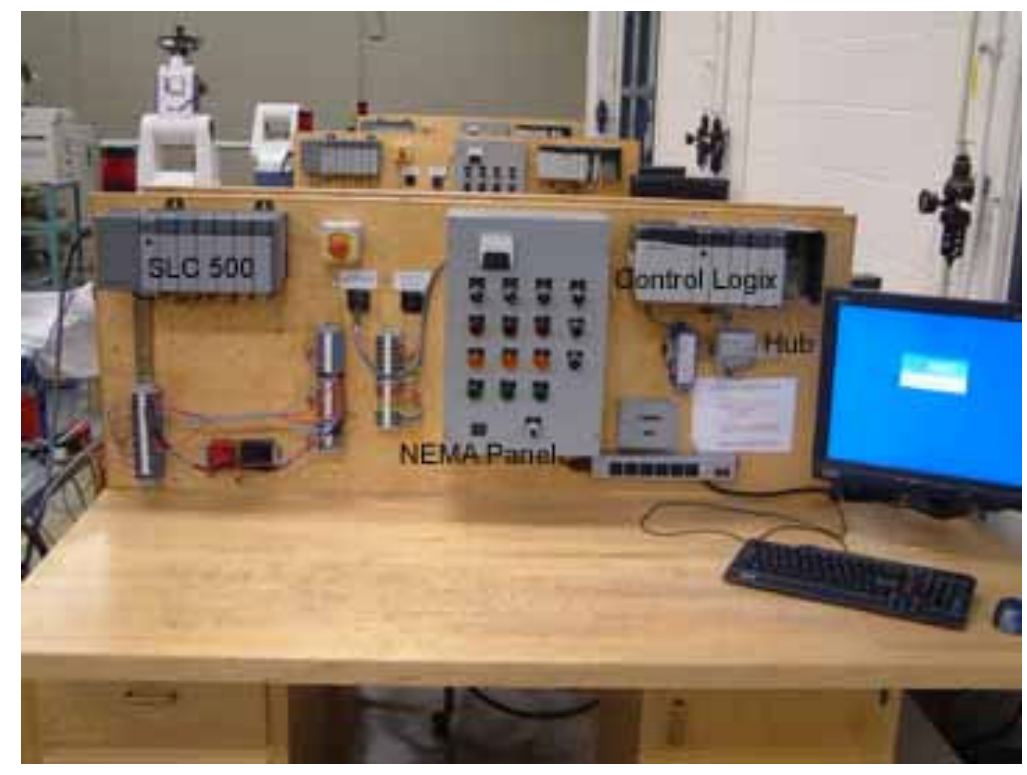

Figure 1

EMET 430 Programmable Logic Controllers II - 3 credits (1 hour lecture and 4 hours laboratory)

- Introduce students to the program structure and IEC 61131 programming languages in the Allen Bradley RS Logix 5000 language on a Control Logix PLC system

- $\quad$ Cover the PLC command structure using each of the 61131 languages with both discrete and analog inputs and outputs

- $\quad$ Execute programs using a manufacturing assembly system that simulates many of the common discrete and analog control requirements found in industry (see Figure 2 for view of assembly system)

- Use a DeviceNet control network to interface the PLC with the automated assembly system

- Use a motion and position control trainer to introduce servo position and speed control industrial applications

- Man-machine interface software (Wonderware or RS View) is used to build an operator interface for the automated assembly system 
The first course is taken in the second or third year and the second course in the sequence is taken in the fall of the senior year.

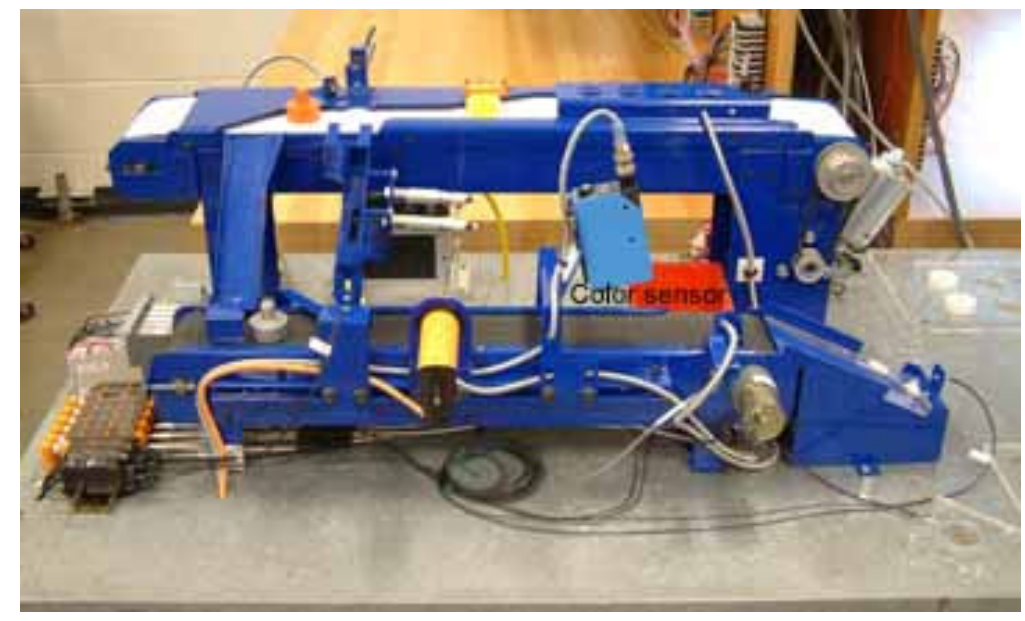

Figure 2

\section{Laboratory Hardware, Network, and Software}

The laboratory for the two PLC courses has eight student stations and supports a laboratory section size of 16 students. There are four assembly systems, (see Figure 2) with each assembly system interfaced with two back-to-back student stations. The laboratory student stations, pictured in Figure 1, have two models of Allen Bradley PLCs: SLC and Control Logix. The SLC 500 system has a 505 processor, standard discrete DC input ant output modules, and an analog I/O module. The Control Logix model has an L55 processor, standard discrete DC input and output modules, and a DeviceNet scanner modules that links to Block I/O and a pneumatic valve block from Festo. The Block I/O has discrete input and output modules and an analog I/O module. A layout of the network for two of the student stations is illustrated in Figure 3.

The network layout shows station A and station B, which are back to back student stations that share a common assembly system, Figure 1, used for programming problems. The PLCs and computers are linked by a local Ethernet LAN that is connected to a server that supports the automation laboratory. A second DeviceNet network links the $\mathrm{I} / \mathrm{O}$ from the assembly system to the Control Logix system. Note that a switch is used to direct which student station has the DeviceNet network attached to the assembly system. The DeviceNet includes a valve control block and Block I/O with discrete and analog inputs and outputs.

The operator switch and pilot lamp panel has a cable and plug (see Figure 1) that is used to connect the panel devices to a set of SLC $500 \mathrm{I} / \mathrm{O}$ or to a set of terminal blocks. The terminal block also interfaces to other SLC $500 \mathrm{I} / \mathrm{O}$ so students can wire a PLC interface for a specific application. Other items illustrated on the network diagram are labeled in Figure 1 as well.

The assembly station, Figure 1, is a modified LabVolt system that assembles a ring on the pin of 
a round base part. Bases are three colors and rings are either white or yellow. The system has three distinct programming problems:

- Parts sorter - bases and rings are introduced into the system in any order and any color. The rings must be ejected from the top conveyor into a queue and the bases must be passed to a deliver chute that drops them to the lower conveyor.

- Queue and assembler - the queue holds four parts with one in the assembly fixture and three above queued for delivery. After the ring in the assembly fixture is placed on top of a base, the queue delivery process must release a new ring to the assembly fixture and cycle the remaining rings down in the queue.

- Quality inspection system - the inspection system must determine that complete assembly is present and that the proper color for the base and ring are present. Good assemblies are passed to the finish part queue and incomplete or incorrect color combinations are ejected into a recycle parts bin.

Nine discrete sensors and one analog color sensor are used to collect production data. Sensors types include inductive and capacitive proximity and photoelectric diffused. The two part stoppers and two part ejectors are either single or double acting pneumatic actuators.

The assembly system offers a number of programming challenges that mirror situations commonly found in industrial automation. Students are required to program the three areas using standard ladder logic and one or more of the new 61131 languages. The Control Logix system and the RS Logix 5000 software is used with the switch and pilot lamp panel and on the assembly system in the second PLC course. The first course uses the SLC 500 system and the switch and pilot lamp panel for program development

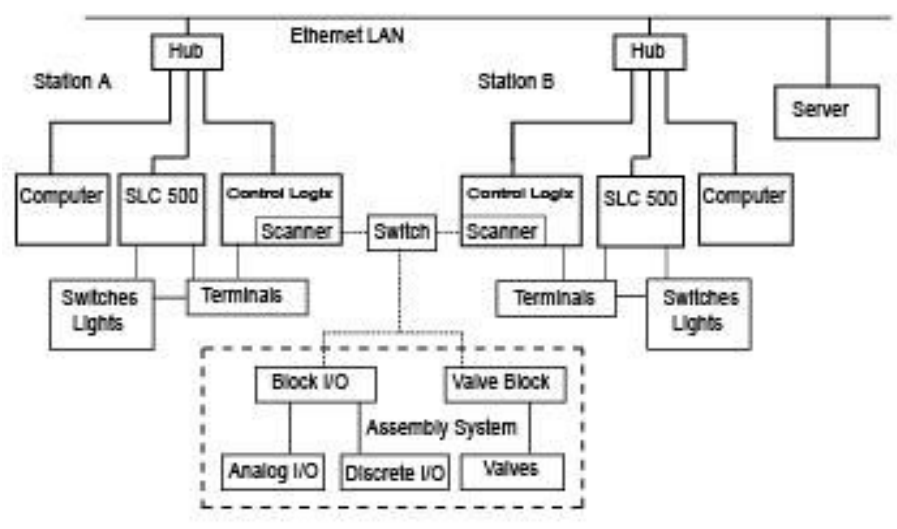

Figure 3

\section{Programming with the IEC 61131 Languages - Goals}

As a result of the laboratory modifications including the addition of the newer Control Logix PLC systems, four of the five new IEC 61131 PLC standard languages were integrated into the second PLC course EMET $430^{2}$. The goal was to give the students an opportunity to solve discrete automation system problems using these four standard languages.

Exercises - Three automation problems were assigned to the students.

Basic: The students were to use a standard NEMA panel and enclosure with momentary push 
buttons, toggle switches, and groups of lights with three different colors. The objective was to write a basic program to activate one of the lights with a momentary push button. This exercise gave the students experience in making electrical connections with the PLC discrete point I/O. It also covered basic addressing and the language command structure.

Robot Simulation: With an understanding of addressing, language command structure, and electrical connections, the students simulated a standard robotic material handling process. In this process, switches on the NEMA panel simulated robotic sensor inputs and lights simulated robotic arm articulation and gripper closure. The students performed the appropriate wiring connections and software solutions to accomplish the manufacturing objective of moving a part from an input hopper to a finished part bin. The goal of this exercise was to use a systematic approach in executing sequential control of a discrete automation system.

Advanced Manufacturing Solution: Having mastered the basics of point I/O addressing, language command structure, and electrical connections, and having solved a structured robot simulation problem using a systematic approach, the students solved an open ended, advanced manufacturing assembly system that mirrored many of the common discrete and analog control requirements found in industry. The functional requirements of the assembly system included:

- Material handling - palletized parts were transferred to a conveyor using an anthropomorphic ac servo drive robot.

- Sorting - sensors were used to sort multicolored plastic rings from multicolored aluminum pegs. The rings were stored in a queue to be prepared for assembly. The queue had an upper limit to the number of rings it could hold. Quantities higher than this limit were to be sent on.

- Assembly - the rings and pegs were assembled.

- Quality control - an analog grey scale color sensor was used for determining pass/fail standards. A standard for quality was to be established by each student team. This involved choosing a color for the base and ring that was considered a good part. The student used integer values taken from the analog grey-scale sensor

While the first two exercises used standard point $\mathrm{I} / \mathrm{O}$, this exercise required the use of a DeviceNet control network to interface the PLC with the automated assembly system. DeviceNet control network $\mathrm{I} / \mathrm{O}$ address mapping and the creation of alias program tags were accomplished in this exercise.

\section{IEC 61131 PLC Standard Programming Languages for Programmable Controllers}

The programming exercises described above gave students an opportunity to learn four of the five IEC 61131 PLC Standard Programming Languages for Programmable Controllers in a controlled environment. Using different programming languages to solve the same problem set brought out the distinctive nature of each language ${ }^{3}$.

\section{Sequential Ladder logic}


Ladder diagrams (LD) or "ladder logic" is the first of the IEC 61131 PLC Standard Programming Languages. It is the most widely used in industry and in most colleges and universities. In the first Penn State Altoona PLC course students became familiar with the Allen Bradley RS Logix 500 programming environment. This program uses only ladder logic and does not support the other IEC 61131 PLC languages. In the second Penn State Altoona PLC course students use the Allen Bradley RS Logix 5000 languages on a Control Logix PLC system. In order to make the transition, students were asked to perform the first two exercises (basic and robot simulation described above) using the familiar Allen Bradley RS Logix 500 programming environment. Following the successful completion of these first two exercises, the students then learned the Allen Bradley RS Logix 5000 ladder logic language on a Control Logix PLC system. They performed the first two exercises again with the new system. A typical rung from a sequential ladder logic program is shown below.

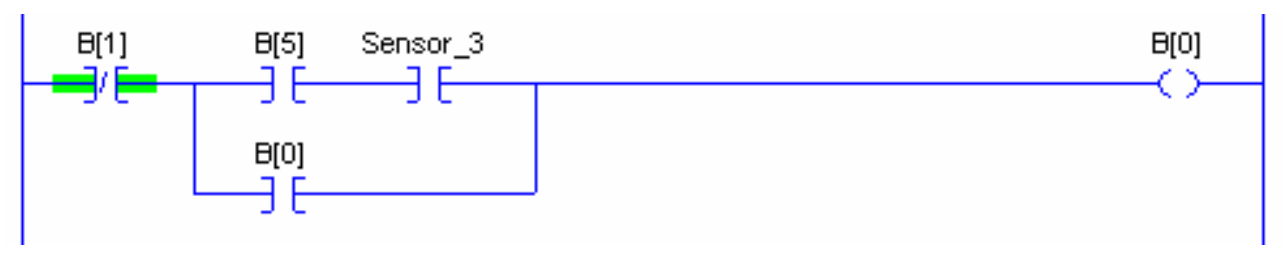

Having mastered the basics of Allen Bradley RS Logix 5000 sequential ladder logic programming and understanding the DeviceNet control network interface to the PLC, the student was then given the task of completing the third exercise using the advanced manufacturing assembly system.

Programming using sequential ladder logic was successfully completed but there was a significant learning curve for the students. The rung structure was clumsy and the maintenance of tag names for each of the instructions proved to be awkward.

\section{Sequential Function Chart (SFC)}

Students were asked to complete the basic exercise for SFC programming. In a short time, successful programs were created and executed. Students then proceeded to complete the second and third exercises using SFC programming. A sample routine is shown below.

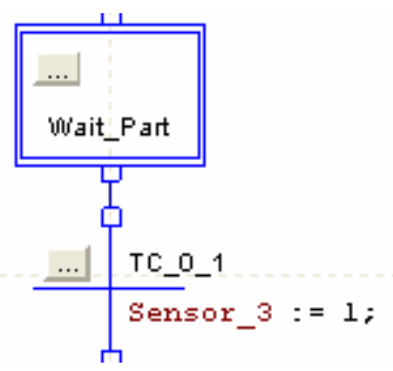

In the third exercise, the advanced manufacturing assembly system, the students completed a successful solution in a much shorter time.

\section{Structured Text}

"Proceedings of the 2005 American Society for Engineering Education Annual Conference \& Exposition Copyright $\square$ 2005, American Society for Engineering Education” 
Students were then asked to complete all three exercises using structured text programming. A sample program section is shown below:

$$
(* * * * * \text { Check for axis on and Gear } * * * * *)
$$

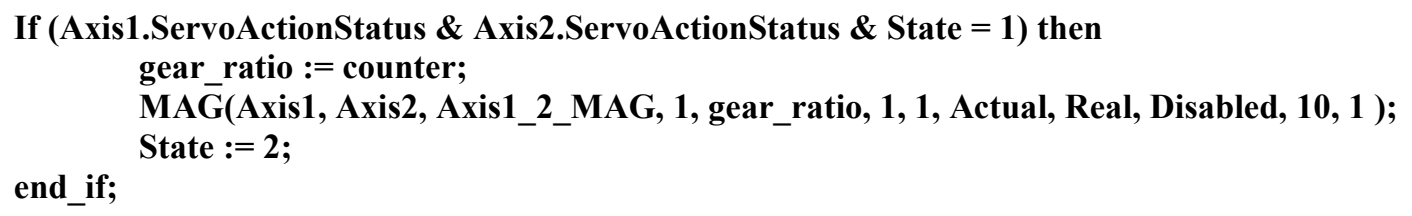

Program solutions were easily created and executed for all three exercises.

\section{Function Block Programming}

Finally, students used function block programming to solve the three programming exercises. A sample FBD is shown below:

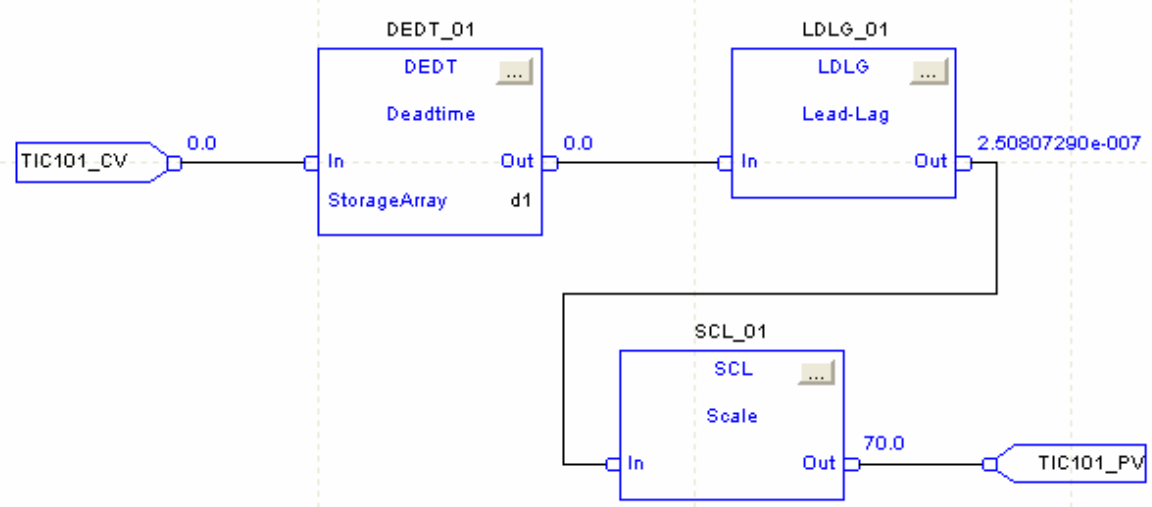

Function block programming solutions were created and students were able to accomplish all of the goals for the advanced manufacturing assembly system.

\section{References:}

1. "IEC 61131 Standard," American National Standards Institute, Inc., New York, NY 10036

2. "General Language Reference Manual 1756-rm003g-en-p," Allen Bradley, Milwaukee, WI, June, 2003.

3. "Process control and drives instruction 1756-rm003c-en-p," Allen Bradley, Milwaukee, WI, June, 2003. 
James A. Rehg - Professor Rehg is an Associate Professor of Engineering at Penn State Altoona and teaches courses in the Electromechanical Engineering Technology 4-year degree program.

Bruce Muller - Professor Muller is a Senior Instructor of Engineering at Penn State Altoona and teaches courses engineering design and graphics and in the Electromechanical Engineering Technology 4-year degree program. 